\title{
A Mixed Monotone Operator Method for the Existence and Uniqueness of Positive Solutions to Impulsive Caputo Fractional Differential Equations
}

\author{
Jieming Zhang, ${ }^{1}$ Chen Yang, ${ }^{1}$ and Chengbo $\mathrm{Zhai}^{2}$ \\ ${ }^{1}$ Basic Course Department, Business College of Shanxi University, Taiyuan, Shanxi 030031, China \\ ${ }^{2}$ School of Mathematical Sciences, Shanxi University, Taiyuan, Shanxi 030006, China \\ Correspondence should be addressed to Chengbo Zhai; cbzhai@sxu.edu.cn
}

Received 10 July 2013; Accepted 8 October 2013

Academic Editor: Jehad Alzabut

Copyright (C) 2013 Jieming Zhang et al. This is an open access article distributed under the Creative Commons Attribution License, which permits unrestricted use, distribution, and reproduction in any medium, provided the original work is properly cited.

We establish some sufficient conditions for the existence and uniqueness of positive solutions to a class of initial value problem for impulsive fractional differential equations involving the Caputo fractional derivative. Our analysis relies on a fixed point theorem for mixed monotone operators. Our result can not only guarantee the existence of a unique positive solution but also be applied to construct an iterative scheme for approximating it. An example is given to illustrate our main result.

\section{Introduction}

The purpose of this paper is to investigate the existence and uniqueness of positive solutions to the following impulsive initial value problem (IVP for short) for Caputo fractionalorder differential equations:

$$
\begin{array}{r}
{ }^{c} D^{\alpha} y(t)=f(t, y(t), y(t))+g(t, y(t)), \\
t \in[0, T], t \neq t_{k}, \\
\left.\Delta y\right|_{t=t_{k}}=I_{k}\left(y\left(t_{k}^{-}\right)\right), \quad y(0)=y_{0},
\end{array}
$$

where $k=1,2, \ldots, m, 0<\alpha \leq 1,{ }^{c} D^{\alpha}$ is the Caputo fractional derivative, $f:[0, T] \times \mathbf{R} \times \mathbf{R} \rightarrow \mathbf{R}$ and $g:[0, T] \times \mathbf{R} \rightarrow \mathbf{R}$ are given functions, $I_{k}: \mathbf{R} \rightarrow \mathbf{R}, y_{0} \in \mathbf{R}, 0=t_{0}<t_{1}<\cdots<$ $t_{m}<t_{m+1}=T,\left.\Delta y\right|_{t=t_{k}}=y\left(t_{k}^{+}\right)-y\left(t_{k}^{-}\right)$, and $y\left(t_{k}^{+}\right)=$ $\lim _{h \rightarrow 0^{+}} y\left(t_{k}+h\right)$ and $y\left(t_{k}^{-}\right)=\lim _{h \rightarrow 0^{-}} y\left(t_{k}+h\right)$ represent the right and left limits of $y(t)$ at $t=t_{k}$.

Fractional differential equations arise in many fields, such as physics, mechanics, chemistry, economics, engineering, and biological sciences; see [1-6], for example. In the recent years, there has been a significant development in ordinary and partial differential equations involving fractional derivatives; see the monographs of Miller and Ross [3], Podlubny
[5], Kilbas et al. [6], and the papers [7-21] and the references therein. In these papers, many authors have investigated the existence of positive solutions for nonlinear fractional differential equation boundary value problems. On the other hand, the uniqueness of positive solutions for nonlinear fractional differential equation boundary value problems has been studied by some authors; see [10, 15, 19, 22, 23], for example.

In [7], Ahmad and Sivasundaram considered the following impulsive hybrid boundary value problem for nonlinear fractional differential equations:

$$
\begin{gathered}
{ }^{c} D^{q} x(t)=f(t, x(t)), \quad 1<q \leq 2, \\
t \in[0,1] \backslash\left\{t_{1}, t_{2}, \ldots, t_{p}\right\}, \\
\Delta x\left(t_{k}\right)=I_{k}\left(x\left(t_{k}^{-}\right)\right), \\
\Delta x^{\prime}\left(t_{k}\right)=J_{k}\left(x\left(t_{k}^{-}\right)\right), \quad t_{k} \in(0,1), k=1,2, \ldots, p, \\
x(0)+x^{\prime}(0)=0, \quad x(1)+x^{\prime}(1)=0,
\end{gathered}
$$

where ${ }^{c} D^{q}$ is the Caputo fractional derivative, $f:[0,1] \times$ $\mathbf{R} \rightarrow \mathbf{R}$ is a continuous function, $I_{k}, J_{k}: \mathbf{R} \rightarrow \mathbf{R}$, $\Delta x\left(t_{k}\right)=x\left(t_{k}^{+}\right)-x\left(t_{k}^{-}\right)$with $x\left(t_{k}^{+}\right)=\lim _{h \rightarrow 0^{+}} x\left(t_{k}+h\right)$, and 
$x\left(t_{k}^{-}\right)=\lim _{h \rightarrow 0^{-}} x\left(t_{k}+h\right), k=1,2, \ldots, p$ for $0=t_{0}<$ $t_{1}<t_{2}<\cdots<t_{p}<t_{p+1}=1$. Based on contraction mapping principle and the Krasnoselskii fixed point theorem, they discussed some existence results for (2).

In [24], Benchohra and Slimani concerned the existence and uniqueness of solutions for the following initial value problem for Caputo fractional-order differential equations:

$$
\begin{gathered}
{ }^{c} D^{\alpha} y(t)=f(t, y(t)), \quad t \in J=[0, T], t \neq t_{k}, \\
\left.\Delta y\right|_{t=t_{k}}=I_{k}\left(y\left(t_{k}^{-}\right)\right), \quad y(0)=y_{0},
\end{gathered}
$$

where $k=1, \ldots, m, 0<\alpha \leq 1, f:[0, T] \times \mathbf{R} \rightarrow \mathbf{R}$ is a given function, $I_{k}: \mathbf{R} \rightarrow \mathbf{R}, y_{0} \in \mathbf{R}, 0=t_{0}<t_{1}<\cdots<$ $t_{m}<t_{m+1}=T,\left.\Delta y\right|_{t=t_{k}}=y\left(t_{k}^{+}\right)-y\left(t_{k}^{-}\right)$, and $y\left(t_{k}^{+}\right)=$ $\lim _{h \rightarrow 0^{+}} y\left(t_{k}+h\right)$ and $y\left(t_{k}^{-}\right)=\lim _{h \rightarrow 0^{-}} y\left(t_{k}+h\right)$ represent the right and left limits of $y(t)$ at $t=t_{k}$. They gave an existence and uniqueness result for the IVP (3) which was based on the Banach fixed point theorem and also obtained two existence and uniqueness results; the first one was based on the Schaefer fixed point theorem and the second one was based on the nonlinear alternative of the Leray-Schauder type.

Different from the above works $[7,24]$, in this paper, we will use a fixed point theorem for mixed monotone operators to study the existence and uniqueness of positive solutions for the IVP (1). Our result can not only guarantee the existence of unique positive solution but also be applied to construct iterative scheme for approximating it.

With this context in mind, the outline of this paper is as follows. In Section 2, we will recall certain results from the theory of fractional calculus and some definitions, notations, and results of mixed monotone operators. In Section 3, we will provide some conditions under which the IVP (1) will have a unique positive solution. Finally, in Section 4, we will provide one example, which explicates the applicability of our main result.

\section{Preliminaries}

For the convenience of the reader, we present here some definitions, lemmas, and basic results that will be used in the proof of our main theorem.

Definition 1 (see [9]). Let $v>0$ with $v \in \mathbf{R}$. Suppose that $y:[a, \infty) \rightarrow \mathbf{R}$. Then the $v$ th Riemann-Liouville fractional integral is defined to be

$$
D_{a^{+}}^{-v} y(t):=\frac{1}{\Gamma(\nu)} \int_{a}^{t} y(s)(t-s)^{\nu-1} d s,
$$

whenever the right-hand side is defined. Similarly, with $v>0$ and $v \in \mathbf{R}$, one defines the $v$ th Riemann-Liouville fractional derivative to be

$$
D_{a^{+}}^{v} y(t):=\frac{1}{\Gamma(n-v)} \frac{d^{n}}{d t^{n}} \int_{a}^{t} \frac{y(s)}{(t-s)^{\nu+1-n}} d s,
$$

where $n \in N$ is the unique positive integer satisfying $n-1 \leq$ $v<n$ and $t>a$.
Definition 2 (see [11]). For a function $y$ given on the interval $[a, b]$, the Caputo fractional-order derivative of order $v$ of $y$ is defined by

$$
{ }^{c} D_{a^{+}}^{\nu} y(t):=\frac{1}{\Gamma(n-\nu)} \int_{a}^{t} \frac{y^{(n)}(s)}{(t-s)^{\nu+1-n}} d s,
$$

where $n=[\nu]+1$.

In the sequel, we present some basic concepts in the ordered Banach spaces for completeness and one fixed point theorem which will be used later. For convenience of readers, we suggest that one refers to $[23,25,26]$ for details.

Suppose that $(E,\|\cdot\|)$ is a real Banach space which is partially ordered by a cone $P \subset E$. That is, $x \leq y$ if and only if $y-x \in P$. If $x \leq y$ and $x \neq y$, then we denote that $x<y$ or $y>x$. By $\theta$ we denote the zero element of $E$. Recall that a nonempty closed convex set $P \subset E$ is a cone if it satisfies (i) $x \in P, \lambda \geq 0 \Rightarrow \lambda x \in P$; (ii) $x \in P,-x \in P \Rightarrow x=\theta$.

$P$ is called normal if there exists a constant $N>0$ such that, for all $x, y \in E, \theta \leq x \leq y$ implies that $\|x\| \leq N\|y\|$; in this case $N$ is called the normality constant of $P$. If $x_{1}, x_{2} \in E$, the set $\left[x_{1}, x_{2}\right]=\left\{x \in E \mid x_{1} \leq x \leq x_{2}\right\}$ is called the order interval between $x_{1}$ and $x_{2}$.

Definition 3 (see $[23,25,26])$. Consider that $A: P \times P \rightarrow P$ is said to be a mixed monotone operator if $A(x, y)$ is increasing in $x$ and decreasing in $y$; that is, $u_{i}, v_{i}(i=1,2) \in P, u_{1} \leq u_{2}$, and $v_{1} \geq v_{2}$ imply that $A\left(u_{1}, v_{1}\right) \leq A\left(u_{2}, v_{2}\right)$. Element $x \in P$ is called a fixed point of $A$ if $A(x, x)=x$.

Lemma 4 (see [25]). Let $P$ be normal and let $A: P \times P \rightarrow P$ be a mixed monotone operator. Suppose that

(a) there exist $v>\theta$ and $c>0$, such that $\theta<A(v, \theta) \leq v$ and $A(\theta, v) \geq c A(v, \theta)$,

(b) for any $0<a<b<1$, there exists $\eta=\eta(a, b)>0$, such that

$$
\begin{array}{r}
A\left(t x, t^{-1} y\right) \geq t(1+\eta) A(x, y), \\
\forall a \leq t \leq b, \quad \theta \leq y \leq x \leq v .
\end{array}
$$

Then $A$ has a unique fixed point $x^{*}$ in $[\theta, v]$ with $x^{*}>\theta$. Moreover, for any initial values $x_{0}, y_{0} \in[\theta, v]$, constructing successively the sequences

$x_{n}=A\left(x_{n-1}, y_{n-1}\right), \quad y_{n}=A\left(y_{n-1}, x_{n-1}\right), \quad n=1,2, \ldots$,

one has $x_{n} \rightarrow x^{*}$ and $y_{n} \rightarrow x^{*}$ as $n \rightarrow \infty$.

\section{Main Result}

In this section, we apply Lemma 4 to study the IVP (1) and then we obtain a new result on the existence and uniqueness of positive solutions. The existence and uniqueness result is relatively new to the fractional differential equations in this literature.

In our considerations we will work in the Banach space $C[0, T]=\{x:[0, T] \rightarrow \mathbf{R}$ is continuous $\}$ with the standard 
norm $\|x\|=\sup \{|x(t)|: t \in[0, T]\}$. Notice that this space can be equipped with a partial order given by

$$
x, y \in C[0, T], \quad x \leq y \Longleftrightarrow x(t) \leq y(t) \text { for } t \in[0, T] \text {. }
$$

Set $P=\{x \in C[0, T] \mid x(t) \geq 0, t \in[0, T]\}$, the standard cone. It is clear that $P$ is a normal cone in $C[0, T]$ and the normality constant is 1 . Consider the following set of functions:

$P C[0, T]$

$$
\begin{aligned}
= & \left\{y:[0, T] \longrightarrow \mathbf{R}: y \in C\left(t_{k}, t_{k+1}\right],\right. \\
& k=0,1, \ldots, m \text { and there exist } y\left(t_{k}^{-}\right) \text {and } y\left(t_{k}^{+}\right), \\
& \left.k=1,2, \ldots, m \text { with } y\left(t_{k}^{-}\right)=y\left(t_{k}\right)\right\} .
\end{aligned}
$$

Then $P C[0, T]$ is a Banach space with the norm

$$
\|y\|_{P C}=\sup _{t \in[0, T]}|y(t)|
$$

Denote $P_{C}$ by $P_{C}=\{x \in P C[0, T] \mid x(t) \geq 0, t \in[0, T]\}$; then $P_{C}$ is a standard and normal cone in $P C[0, T]$.

Lemma 5 (see [24]). Let $0<\alpha \leq 1$ and let $h: J \rightarrow \mathbf{R}$ be continuous. A function $y \in P C[0, T]$ is a solution of the fractional integral equation:

$$
y(t)=\left\{\begin{array}{rlr}
y_{0}+\frac{1}{\Gamma(\alpha)} \int_{0}^{t}(t-s)^{\alpha-1} h(s) d s, & \text { if } t \in\left[0, t_{1}\right], \\
y_{0}+\frac{1}{\Gamma(\alpha)} \sum_{i=1}^{k} \int_{t_{i-1}}^{t_{i}}\left(t_{i}-s\right)^{\alpha-1} h(s) d s & \\
& +\frac{1}{\Gamma(\alpha)} \int_{t_{k}}^{t}(t-s)^{\alpha-1} h(s) d s & \\
& +\sum_{i=1}^{k} I_{i}\left(y\left(t_{i}^{-}\right)\right) & \text {if } t \in\left(t_{k}, t_{k+1}\right]
\end{array}\right.
$$

where $k=1, \ldots, m$, if and only if $y$ is a solution of the fractional IVP

$$
\begin{gathered}
{ }^{c} D^{\alpha} y(t)=h(t), \quad t \in[0, T], t \neq t_{k}, \\
\left.\Delta y\right|_{t=t_{k}}=I_{k}\left(y\left(t_{k}^{-}\right)\right), \quad k=1, \ldots, m, \\
y(0)=y_{0} .
\end{gathered}
$$

Remark 6. The concept of solutions for fractional differential equations has been argued extensively; see [7, 18, 21, 24] and the references therein. In [18], the authors gave a counterexample to show that the result in Lemma 5 is not reasonable. However, the counterexample is inappropriate. From the very recent paper [21], the approach for finding the solution of impulsive fractional differential equations in [18] is inappropriate and some arguments like Lemma 5 are plausible.
For the convenience, we list the following conditions:

$\left(H_{1}\right) f:[0, T] \times[0, \infty) \times[0, \infty) \rightarrow[0, \infty)$ is continuous and $g:[0, T] \times[0, \infty) \rightarrow[0, \infty)$ is continuous;

$\left(H_{2}\right) f(t, u, v)$ is increasing in $u \in[0, \infty)$ for fixed $t \in[0, T]$ and $v \in[0, \infty)$ and decreasing in $v \in[0, \infty)$ for fixed $t \in[0, T]$ and $u \in[0, \infty)$, and $g(t, v)$ is decreasing in $v \in[0, \infty)$ for fixed $t \in[0, T]$;

$\left(H_{3}\right)$ for any $0<p<q<1$, there exist $\beta_{1}, \beta_{2}$, and $\beta_{3} \in$ $(0, \infty)$ which depend on $q$, such that

$$
f\left(t, \lambda u, \lambda^{-1} v\right) \geq \frac{\lambda}{1-\lambda^{\beta_{1}}} f(t, u, v),
$$

$\forall t \in[0, T], \quad p \leq \lambda \leq q, u, v \in[0, \infty)$,

$$
g\left(t, \lambda^{-1} v\right) \geq \frac{\lambda}{1-\lambda^{\beta_{2}}} g(t, v)
$$

$\forall t \in[0, T], p \leq \lambda \leq q, v \in[0, \infty)$

$$
\begin{aligned}
& i I_{k}(\lambda u) \geq \frac{\lambda}{1-\lambda^{\beta_{3}}} I_{k}(u), \\
& \forall k=1,2, \ldots m, p \leq \lambda \leq q, u \in[0, \infty) ;
\end{aligned}
$$

$\left(H_{4}\right)$ the functions $I_{k}:[0, \infty) \rightarrow \mathbf{R}$ are continuous and nondecreasing and there exists a constant $M^{*}>0$ such that $\left|I_{k}(u)\right| \leq M^{*}$ for each $u \in[0, \infty), k=$ $1, \ldots, m$;

$\left(H_{5}\right) y_{0}>m M^{*}>0$.

Theorem 7. Assume that $\left(H_{1}\right)-\left(H_{5}\right)$ are satisfied and there exists $R>0$ such that

$$
y_{0}+\frac{(m+1)\left(M_{1}+M_{2}\right)}{\Gamma(\alpha+1)} T^{\alpha}+m M^{*} \leq R,
$$

where $M_{1}=\max _{t \in[0, T]} f(t, R, 0), M_{2}=\max _{t \in[0, T]} g(t, 0)$.

Then the IVP (1) has a unique positive solution $u^{*}$ in $P_{[\theta, R]}=\left\{y \in P_{C} \mid 0 \leq y(t) \leq R, t \in[0, T]\right\}$ with $u^{*}(t) \not \equiv 0$, $t \in[0, T]$. Moreover, for any initial values $x_{0}, y_{0} \in P_{C}$, constructing successively the sequences

$$
\begin{aligned}
& x_{n+1}(t) \\
& =y_{0}+\frac{1}{\Gamma(\alpha)} \\
& \quad \times \sum_{0<t_{k}<t} \int_{t_{k-1}}^{t_{k}}\left(t_{k}-s\right)^{\alpha-1}\left[f\left(s, x_{n}(s), y_{n}(s)\right)\right. \\
& +\frac{1}{\Gamma(\alpha)} \int_{t_{k}}^{t}(t-s)^{\alpha-1}\left[f\left(s, y_{n}(s)\right)\right] d s \\
& \left.\quad+g\left(s, y_{n}(s)\right)\right] d s \\
& +\sum_{0<t_{k}<t} I_{k}\left(x_{n}\left(t_{k}^{-}\right)\right),
\end{aligned}
$$




$$
\begin{aligned}
y_{n+1}(t)= & y_{0}+\frac{1}{\Gamma(\alpha)} \\
& \times \sum_{0<t_{k}<t} \int_{t_{k-1}}^{t_{k}}\left(t_{k}-s\right)^{\alpha-1}\left[f\left(s, y_{n}(s), x_{n}(s)\right)\right. \\
& \left.+g\left(s, x_{n}(s)\right)\right] d s \\
& +\frac{1}{\Gamma(\alpha)} \int_{t_{k}}^{t}(t-s)^{\alpha-1}\left[f\left(s, y_{n}(s), x_{n}(s)\right)\right. \\
& +\sum_{0<t_{k}<t} I_{k}\left(y_{n}\left(t_{k}^{-}\right)\right),
\end{aligned}
$$

where $n=0,1,2, \ldots$, then one has $\left\|x_{n}-u^{*}\right\| \rightarrow 0$ and $\| y_{n}$ $u^{*} \| \rightarrow 0$ as $n \rightarrow \infty$.

Proof. To begin with, from Lemma 5, the IVP (1) has an integral formulation given by

$$
\begin{aligned}
u(t)= & y_{0}+\frac{1}{\Gamma(\alpha)} \\
& \times \sum_{0<t_{k}<t} \int_{t_{k-1}}^{t_{k}}\left(t_{k}-s\right)^{\alpha-1}[f(s, u(s), u(s)) \\
& +g(s, u(s))] d s \\
& +\frac{1}{\Gamma(\alpha)} \int_{t_{k}}^{t}(t-s)^{\alpha-1}[f(s, u(s), u(s)) \\
& +\sum_{0<t_{k}<t} I_{k}\left(u\left(t_{k}^{-}\right)\right) .
\end{aligned}
$$

Define an operator $A: P_{C} \times P_{C} \rightarrow P C[0, T]$ by

$$
\begin{aligned}
A(u, v)(t)= & y_{0}+\frac{1}{\Gamma(\alpha)} \\
& \times \sum_{0<t_{k}<t} \int_{t_{k-1}}^{t_{k}}\left(t_{k}-s\right)^{\alpha-1}[f(s, u(s), v(s)) \\
& +g(s, v(s))] d s \\
& +\frac{1}{\Gamma(\alpha)} \int_{t_{k}}^{t}(t-s)^{\alpha-1}[f(s, u(s), v(s)) \\
& +\sum_{0<t_{k}<t} I_{k}\left(u\left(t_{k}^{-}\right)\right) .
\end{aligned}
$$

It is easy to prove that $u$ is the solution of the IVP (1) if and only if $u$ is the fixed point of $A$. From $\left(H_{1}\right),\left(H_{4}\right)$, and $\left(H_{5}\right)$, we know that

$$
\begin{aligned}
A(u, v)(t)= & y_{0}+\frac{1}{\Gamma(\alpha)} \\
& \times \sum_{0<t_{k}<t} \int_{t_{k-1}}^{t_{k}}\left(t_{k}-s\right)^{\alpha-1}[f(s, u(s), v(s)) \\
& +g(s, v(s))] d s \\
& +\frac{1}{\Gamma(\alpha)} \int_{t_{k}}^{t}(t-s)^{\alpha-1}[f(s, u(s), v(s)) \\
& +\sum_{0<t_{k}<t} I_{k}\left(u\left(t_{k}^{-}\right)\right), \\
\geq & y_{0}-m M^{*}>0,
\end{aligned}
$$

for any $u, v \in P_{C}, t \in[0, T]$. So, $A: P_{C} \times P_{C} \rightarrow P_{C}$. In the sequel we check that $A$ satisfies all assumptions of Lemma 4 .

Firstly, we prove that $A$ is a mixed monotone operator. In fact, for $u_{i}, v_{i} \in P_{C}, i=1,2$ with $u_{1} \geq u_{2}, v_{1} \leq v_{2}$, we know that $u_{1}(t) \geq u_{2}(t), v_{1}(t) \leq v_{2}(t)$, and $t \in[0, T]$, and by $\left(H_{2}\right)$ and Definition 3, we have that

$$
\begin{aligned}
& A\left(u_{1}, v_{1}\right)(t)= y_{0}+\frac{1}{\Gamma(\alpha)} \\
& \times \sum_{0<t_{k}<t} \int_{t_{k-1}}^{t_{k}}\left(t_{k}-s\right)^{\alpha-1}\left[f\left(s, u_{1}(s), v_{1}(s)\right)\right. \\
&\left.+g\left(s, v_{1}(s)\right)\right] d s \\
&+\frac{1}{\Gamma(\alpha)} \int_{t_{k}}^{t}(t-s)^{\alpha-1}\left[f\left(s, u_{1}(s), v_{1}(s)\right)\right. \\
&\left.+\sum_{0<t_{k}<t} I_{k}\left(u_{1}\left(t_{k}^{-}\right)\right) \quad+g\left(s, v_{1}(s)\right)\right] d s \\
& \geq\left.y_{0}+\frac{1}{\Gamma(\alpha)} \quad+g\left(s, v_{2}(s)\right)\right] d s \\
& \times \sum_{0<t_{k}<t} \int_{t_{k-1}}^{t_{k}}\left(t_{k}-s\right)^{\alpha-1}\left[f\left(s, u_{2}(s), v_{2}(s)\right)\right. \\
&+\frac{1}{\Gamma(\alpha)} \int_{t_{k}}^{t}(t-s)^{\alpha-1}\left[f\left(s, u_{2}(s), v_{2}(s)\right)\right. \\
&\left.+g\left(s, v_{2}(s)\right)\right] d s
\end{aligned}
$$




$$
\begin{aligned}
& \quad+\sum_{0<t_{k}<t} I_{k}\left(u_{2}\left(t_{k}^{-}\right)\right) \\
& =A\left(u_{2}, v_{2}\right)(t) .
\end{aligned}
$$

That is, $A\left(u_{1}, v_{1}\right) \geq A\left(u_{2}, v_{2}\right)$.

Next, we show that $A$ satisfies the condition (a) of Lemma 4. Now, we set $v(t) \equiv R(t \in[0, T])$. Then, $v>\theta$.

On the one hand, from $\left(H_{1}\right)$ and $\left(H_{5}\right)$, we have

$$
\begin{aligned}
& A(v, \theta)(t) \\
& =y_{0}+\frac{1}{\Gamma(\alpha)} \\
& \quad \times \sum_{0<t_{k}<t} \int_{t_{k-1}}^{t_{k}}\left(t_{k}-s\right)^{\alpha-1}[f(s, R, 0)+g(s, 0)] d s \\
& \quad+\frac{1}{\Gamma(\alpha)} \int_{t_{k}}^{t}(t-s)^{\alpha-1}[f(s, R, 0)+g(s, 0)] d s \\
& \quad+\sum_{0<t_{k}<t} I_{k}(R), \\
& \geq y_{0}-m M^{*}>0 .
\end{aligned}
$$

That is to say, $A(v, \theta)>\theta$. From (15), for any $t \in[0, T]$, we obtain

$$
\begin{aligned}
A(v, \theta)(t) \leq & y_{0}+\frac{m\left(M_{1}+M_{2}\right)}{\Gamma(\alpha+1)} T^{\alpha} \\
& +\frac{\left(M_{1}+M_{2}\right)}{\Gamma(\alpha+1)} T^{\alpha}+m M^{*} \\
\leq & R=v(t)
\end{aligned}
$$

That is, $A(v, \theta) \leq v$.

On the other hand, we take $c \in(0,1)$ such that

$$
c \leq \min \left\{\frac{m_{1}+m_{2}}{M_{1}+M_{2}}, \frac{m_{3}}{M^{*}}\right\},
$$

where $m_{1}=\min _{t \in[0, T]} f(t, 0, R), m_{2}=\min _{t \in[0, T]} g(t, R)$, and $m_{3}=\min _{k \in\{1, \ldots, m\}} I_{k}(0)$. For any $t \in[0, T]$,

$$
\begin{aligned}
& A(\theta, v)(t) \\
& =y_{0}+\frac{1}{\Gamma(\alpha)} \\
& \quad \times \sum_{0<t_{k}<t} \int_{t_{k-1}}^{t_{k}}\left(t_{k}-s\right)^{\alpha-1}[f(s, 0, R) \\
& +\frac{1}{\Gamma(\alpha)} \int_{t_{k}}^{t}(t-s)^{\alpha-1}[f(s, 0, R)+g(s, R)] d s \\
& +\sum_{0<t_{k}<t} I_{k}(0)
\end{aligned}
$$

$$
\begin{aligned}
& \geq y_{0}+\frac{1}{\Gamma(\alpha)} \\
& \times \sum_{0<t_{k}<t} \int_{t_{k-1}}^{t_{k}}\left(t_{k}-s\right)^{\alpha-1}\left(m_{1}+m_{2}\right) d s \\
& +\frac{1}{\Gamma(\alpha)} \int_{t_{k}}^{t}(t-s)^{\alpha-1}\left(m_{1}+m_{2}\right) d s \\
& +\sum_{0<t_{k}<t} m_{3} \\
& \geq y_{0}+\frac{1}{\Gamma(\alpha)} \\
& \times \sum_{0<t_{k}<t} \int_{t_{k-1}}^{t_{k}}\left(t_{k}-s\right)^{\alpha-1} c\left(M_{1}+M_{2}\right) d s \\
& +\frac{1}{\Gamma(\alpha)} \int_{t_{k}}^{t}(t-s)^{\alpha-1} c\left(M_{1}+M_{2}\right) d s \\
& +\sum_{0<t_{k}<t} c M^{*} \\
& \geq c y_{0}+\frac{c}{\Gamma(\alpha)} \\
& \times \sum_{0<t_{k}<t} \int_{t_{k-1}}^{t_{k}}\left(t_{k}-s\right)^{\alpha-1}[f(s, R, 0) \\
& +g(s, 0)] d s \\
& +\frac{c}{\Gamma(\alpha)} \int_{t_{k}}^{t}(t-s)^{\alpha-1}[f(s, R, 0)+g(s, 0)] d s \\
& +c \sum_{0<t_{k}<t} I_{k}(R) \\
& =c A(v, \theta)(t) \text {. }
\end{aligned}
$$

That is to say, $A(\theta, v) \geq c A(v, \theta)$. Therefore, condition (a) of Lemma 4 holds.

Finally, we show that $A$ satisfies condition $(b)$ of Lemma 4 . Let $0<a<b<1$ and

$$
\eta=\min \left\{\frac{1}{b}-1, \frac{1}{1-a^{\beta_{1}}}-1, \frac{1}{1-a^{\beta_{2}}}-1, \frac{1}{1-a^{\beta_{3}}}-1\right\}>0 .
$$

For any $a \leq \lambda \leq b, x, y \in P_{C}$, and $t \in[0, \mathrm{~T}]$, by $\left(H_{3}\right)$ we have

$$
\begin{array}{r}
A\left(\lambda x, \lambda^{-1} y\right)(t) \\
=y_{0}+\frac{1}{\Gamma(\alpha)} \\
\quad \times \sum_{0<t_{k}<t} \int_{t_{k-1}}^{t_{k}}\left(t_{k}-s\right)^{\alpha-1}\left[f\left(s, \lambda x(s), \lambda^{-1} y(s)\right)\right. \\
\left.+g\left(s, \lambda^{-1} y(s)\right)\right] d s
\end{array}
$$




$$
\begin{aligned}
& +\frac{1}{\Gamma(\alpha)} \int_{t_{k}}^{t}(t-s)^{\alpha-1}\left[f\left(s, \lambda x(s), \lambda^{-1} y(s)\right)\right. \\
& \left.+g\left(s, \lambda^{-1} y(s)\right)\right] d s \\
& +\sum_{0<t_{k}<t} I_{k}\left(\lambda x\left(t_{k}^{-}\right)\right) \\
& \geq y_{0}+\frac{1}{\Gamma(\alpha)} \\
& \times \sum_{0<t_{k}<t} \int_{t_{k-1}}^{t_{k}}\left(t_{k}-s\right)^{\alpha-1}\left[\frac{\lambda}{1-\lambda^{\beta_{1}}} f(s, x(s), y(s))\right. \\
& \left.+\frac{\lambda}{1-\lambda^{\beta_{2}}} g(s, y(s))\right] d s \\
& +\frac{1}{\Gamma(\alpha)} \int_{t_{k}}^{t}(t-s)^{\alpha-1}\left[\frac{\lambda}{1-\lambda^{\beta_{1}}} f(s, x(s), y(s))\right. \\
& \left.+\frac{\lambda}{1-\lambda^{\beta_{2}}} g(s, y(s))\right] d s \\
& +\sum_{0<t_{k}<t} \frac{\lambda}{1-\lambda^{\beta_{3}}} I_{k}\left(x\left(t_{k}^{-}\right)\right) \\
& \geq y_{0}+\frac{1}{\Gamma(\alpha)} \\
& \times \sum_{0<t_{k}<t} \int_{t_{k-1}}^{t_{k}}\left(t_{k}-s\right)^{\alpha-1}\left[\frac{\lambda}{1-a^{\beta_{1}}} f(s, x(s), y(s))\right. \\
& \left.+\frac{\lambda}{1-a^{\beta_{2}}} g(s, y(s))\right] d s \\
& +\frac{1}{\Gamma(\alpha)} \int_{t_{k}}^{t}(t-s)^{\alpha-1}\left[\frac{\lambda}{1-a^{\beta_{1}}} f(s, x(s), y(s))\right. \\
& \left.+\frac{\lambda}{1-a^{\beta_{2}}} g(s, y(s))\right] d s \\
& +\sum_{0<t_{k}<t} \frac{\lambda}{1-a^{\beta_{3}}} I_{k}\left(x\left(t_{k}^{-}\right)\right) \\
& \geq b(1+\eta) y_{0}+\frac{1}{\Gamma(\alpha)} \\
& \times \sum_{0<t_{k}<t} \int_{t_{k-1}}^{t_{k}}\left(t_{k}-s\right)^{\alpha-1}[\lambda(1+\eta) f(s, x(s), y(s)) \\
& +\lambda(1+\eta) g(s, y(s))] d s \\
& +\frac{1}{\Gamma(\alpha)} \int_{t_{k}}^{t}(t-s)^{\alpha-1}[\lambda(1+\eta) f(s, x(s), y(s)) \\
& +\lambda(1+\eta) g(s, y(s))] d s \\
& +\sum_{0<t_{k}<t} \lambda(1+\eta) I_{k}\left(x\left(t_{k}^{-}\right)\right) \\
& \geq \lambda(1+\eta) A(x, y)(t) \text {. }
\end{aligned}
$$

That is to say, $A\left(\lambda x, \lambda^{-1} y\right) \geq \lambda(1+\eta) A(x, y)$, for all $a \leq \lambda \leq b$, $x, y \in P_{C}$. Hence, condition (b) of Lemma 4 is satisfied. So, the conclusion of Theorem 7 follows from Lemma 4.

\section{An Example}

We present one example to illustrate Theorem 7.

Example 1. Consider the following fractional IVP:

$$
\begin{aligned}
{ }^{c} D^{1 / 5} y(t)= & \tau(t)+\delta(t)+\frac{y(t)}{1+y(t)} \\
& +\frac{2}{1+y(t)}+2, \quad t \in[0,1], t \neq \frac{1}{3}, \\
\left.\Delta y\right|_{t=(1 / 3)}= & \frac{y\left(1 / 3^{-}\right)}{4+y\left(1 / 3^{-}\right)}+1, \quad y(0)=2,
\end{aligned}
$$

where $\tau, \delta \in C([0,1,0, \infty))$.

In this example,

$$
\begin{gathered}
\alpha=\frac{1}{5}, \quad T=1, \quad t_{1}=\frac{1}{3}, \\
m=1, \quad y_{0}=2, \\
f(t, x, y)=\tau(t)+1+\frac{x}{1+x}+\frac{1}{1+y}, \\
g(t, y)=\delta(t)+1+\frac{1}{1+y}, \\
I_{1}(x)=1+\frac{x}{4+x} .
\end{gathered}
$$

Then, $f(t, x, y) \in C([0,1] \times[0, \infty) \times[0, \infty),[0, \infty))$ is continuous and increasing in $x \in[0, \infty)$ for fixed $t \in[0,1]$ and $y \in[0, \infty)$ and decreasing in $y \in[0, \infty)$ for fixed $t \in[0,1]$ and $x \in[0, \infty)$. And $g(t, y) \in C([0,1] \times[0, \infty)$, $[0, \infty))$ is continuous and decreasing in $y \in[0, \infty)$ for fixed $t \in[0,1]$. Moreover, for any $0<p<q<1$, we take

$$
\begin{gathered}
\beta_{1} \geq \log _{q}\left(1-\frac{3 q}{1+2 q}\right), \quad \beta_{2} \geq \log _{q}\left(1-\frac{2 q}{1+q}\right), \\
\beta_{3} \geq \log _{q}\left(1-\frac{2 q}{1+q}\right) .
\end{gathered}
$$

For any $p \leq \lambda \leq q, t \in[0,1]$, and $x, y \in[0, \infty)$, we have

$$
\begin{aligned}
f\left(t, \lambda x, \lambda^{-1} y\right) & =\tau(t)+1+\frac{\lambda x}{1+\lambda x}+\frac{\lambda}{\lambda+y} \\
& \geq \frac{\lambda}{q}(\tau(t)+1)+\lambda\left(\frac{x}{1+x}+\frac{1}{1+y}\right) .
\end{aligned}
$$

We show that

$$
f\left(t, \lambda x, \lambda^{-1} y\right) \geq \frac{\lambda}{1-\lambda^{\beta_{1}}} f(t, x, y),
$$


if and only if we can prove that

$$
\begin{aligned}
& \frac{1}{q}(\tau(t)+1)+\frac{x}{1+x}+\frac{1}{1+y} \\
& \quad \geq \frac{1}{1-\lambda^{\beta_{1}}}\left(\tau(t)+1+\frac{x}{1+x}+\frac{1}{1+y}\right) .
\end{aligned}
$$

That is to say,

$$
\begin{aligned}
(\tau(t) & +1)\left(\frac{1}{q}-\frac{1}{1-\lambda^{\beta_{1}}}\right) \\
& \geq\left(\frac{1}{1-\lambda^{\beta_{1}}}-1\right)\left(\frac{x}{1+x}+\frac{1}{1+y}\right) .
\end{aligned}
$$

From (29), we get

$$
\lambda^{\beta_{1}} \leq q^{\beta_{1}} \leq 1-\frac{3 q}{1+2 q}
$$

and then

$$
\frac{1}{q}-\frac{1}{1-\lambda^{\beta_{1}}} \geq 2\left(\frac{1}{1-\lambda^{\beta_{1}}}-1\right) .
$$

Hence, (33) is satisfied. So, (31) holds.

Similarly, we obtain that

$$
\begin{aligned}
g\left(t, \lambda^{-1} y\right) & =\delta(t)+1+\frac{\lambda}{\lambda+y} \\
& \geq \frac{\lambda}{q}(\delta(t)+1)+\frac{\lambda}{1+y} \\
& \geq \frac{\lambda}{1-\lambda^{\beta_{2}}}\left(\delta(t)+1+\frac{1}{1+y}\right) \\
& =\frac{\lambda}{1-\lambda^{\beta_{2}}} g(t, y), \\
I_{1}(\lambda x) & =1+\frac{\lambda x}{4+\lambda x} \geq \frac{\lambda}{q}+\frac{\lambda x}{4+x} \\
& \geq \frac{\lambda}{1-\lambda^{\beta_{3}}}\left(1+\frac{x}{4+x}\right)=\frac{\lambda}{1-\lambda^{\beta_{3}}} I_{1}(x) .
\end{aligned}
$$

Further, $I_{1}(x)$ is continuous, increasing in $x \in[0, \infty)$, and

$$
\left|I_{1}(x)\right|=\left|\frac{x}{4+x}\right| \leq 1 \equiv M^{*}, \quad \forall x \in[0, \infty) .
$$

And, we also have $y_{0}=2>1=m M^{*}>0$.

Finally, owing to $\tau, \delta \in C([0,1],[0, \infty))$, we have that

$$
\tau^{*}=\max _{t \in[0,1]} \tau(t), \quad \delta^{*}=\max _{t \in[0,1]} \delta(t) .
$$

We take $R>0$ such that

$$
3+\frac{2\left(5+\tau^{*}+\delta^{*}\right)}{\Gamma((1 / 5)+1)} \leq R .
$$

And thus,

$$
\begin{gathered}
M_{1}=\max _{t \in[0,1]} f(t, R, 0) \\
=\max _{t \in[0,1]}\left(\tau(t)+1+\frac{R}{1+R}+1\right) \leq 3+\tau^{*}, \\
M_{2}=\max _{t \in[0,1]} g(t, 0)=\max _{t \in[0,1]}(\delta(t)+1+1)=\delta^{*}+2 .
\end{gathered}
$$

Then,

$$
\begin{aligned}
y_{0}+ & \frac{(m+1)\left(M_{1}+M_{2}\right)}{\Gamma(\alpha+1)} T^{\alpha}+m M^{*} \\
& \leq 2+\frac{2}{\Gamma((1 / 5)+1)}\left(3+\tau^{*}+2+\delta^{*}\right)+1 \\
& =3+\frac{2\left(5+\tau^{*}+\delta^{*}\right)}{\Gamma((1 / 5)+1)} \leq R .
\end{aligned}
$$

Hence, (15) holds. Therefore, all the conditions of Theorem 7 are satisfied. An application of Theorem 7 implies that problem (27) has a unique positive solution.

\section{Acknowledgments}

This research was supported by the Youth Science Foundation of China (11201272), the Science Foundation of Shanxi Province (2013011003-3), and the Science Foundation of Business College of Shanxi University (2012050).

\section{References}

[1] K. B. Oldham and J. Spanier, The Fractional Calculus, vol. 111 of Mathematics in Science and Engineering, Academic Press, New York, NY, USA, 1974.

[2] L. Gaul, P. Klein, and S. Kemple, "Damping description involving fractional operators," Mechanical Systems and Signal Processing, vol. 5, no. 2, pp. 81-88, 1991.

[3] K. S. Miller and B. Ross, An Introduction to the Fractional Calculus and Fractional Differential Equations, John Wiley \& Sons, New York, NY, USA, 1993.

[4] W. G. Glockle and T. F. Nonnenmacher, "A fractional calculus approach to self-similar protein dynamics," Biophysical Journal, vol. 68, no. 1, pp. 46-53, 1995.

[5] I. Podlubny, Fractional Differential Equations, vol. 198 of Mathematics in Science and Engineering, Academic Press, San Diego, Calf, USA, 1999.

[6] A. A. Kilbas, H. M. Srivastava, and J. J. Trujillo, Theory and Applications of Fractional Differential Equations, vol. 204 of North-Holland Mathematics Studies, Elsevier Science B.V., Amsterdam, The Netherlands, 2006.

[7] B. Ahmad and S. Sivasundaram, "Existence results for nonlinear impulsive hybrid boundary value problems involving fractional differential equations," Nonlinear Analysis: Hybrid Systems, vol. 3, no. 3, pp. 251-258, 2009.

[8] Z. Bai and H. Lü, "Positive solutions for boundary value problem of nonlinear fractional differential equation," Journal of Mathematical Analysis and Applications, vol. 311, no. 2, pp. 495-505, 2005. 
[9] C. S. Goodrich, "Existence of a positive solution to a class of fractional differential equations," Applied Mathematics Letters, vol. 23, no. 9, pp. 1050-1055, 2010.

[10] Y. Zhou, "Existence and uniqueness of fractional functional differential equations with unbounded delay," International Journal of Dynamical Systems and Differential Equations, vol. 1, no. 4, pp. 239-244, 2008.

[11] A. A. Kilbas and S. A. Marzan, "Nonlinear differential equations with the Caputo fractional derivative in the space of continuously differentiable functions," Differential Equations, vol. 41, pp. 84-89, 2005.

[12] E. R. Kaufmann and E. Mboumi, "Positive solutions of a boundary value problem for a nonlinear fractional differential equation," Electronic Journal of Qualitative Theory of Differential Equations, vol. 2008, no. 3, pp. 1-11, 2008.

[13] N. Kosmatov, "A singular boundary value problem for nonlinear differential equations of fractional order," Journal of Applied Mathematics and Computing, vol. 29, no. 1-2, pp. 125-135, 2009.

[14] X. Xu, D. Jiang, and C. Yuan, "Multiple positive solutions for the boundary value problem of a nonlinear fractional differential equation," Nonlinear Analysis: Theory, Methods \& Applications, vol. 71, no. 10, pp. 4676-4688, 2009.

[15] L. Yang and H. Chen, "Unique positive solutions for fractional differential equation boundary value problems," Applied Mathematics Letters, vol. 23, no. 9, pp. 1095-1098, 2010.

[16] Y. Wang, L. Liu, and Y. Wu, "Positive solutions for a nonlocal fractional differential equation," Nonlinear Analysis: Theory, Methods \& Applications, vol. 74, no. 11, pp. 3599-3605, 2011.

[17] C. Lizama, "An operator theoretical approach to a class of fractional order differential equations," Applied Mathematics Letters, vol. 24, no. 2, pp. 184-190, 2011.

[18] M. Fečkan, Y. Zhou, and J. Wang, "On the concept and existence of solution for impulsive fractional differential equations," Communications in Nonlinear Science and Numerical Simulation, vol. 17, no. 7, pp. 3050-3060, 2012.

[19] Z. Liu and X. Li, "Existence and uniqueness of solutions for the nonlinear impulsive fractional differential equations," Communications in Nonlinear Science and Numerical Simulation, vol. 18, no. 6, pp. 1362-1373, 2013.

[20] Z. H. Liu, X. W. Li, and I. Sznt, "Existence of solutions for fractional impulsive differential equations with $p$-Laplacian operator," Acta Mathematica Hungarica, vol. 141, no. 3, pp. 203219, 2013.

[21] G. T. Wang, B. Ahmad, L. H. Zhang, and J. J. Nieto, "Comments on the concept of existence of solution for impulsive fractional differential equations," Communications in Nonlinear Science and Numerical Simulation, vol. 19, no. 3, pp. 401-403, 2013.

[22] C. Yang and C. Zhai, "Uniqueness of positive solutions for a fractional differential equation via a fixed point theorem of a sum operator," Electronic Journal of Differential Equations, vol. 2012, no. 70, pp. 1-8, 2012.

[23] C. Zhai and M. Hao, "Fixed point theorems for mixed monotone operators with perturbation and applications to fractional differential equation boundary value problems," Nonlinear Analysis: Theory, Methods \& Applications, vol. 75, no. 4, pp. 2542-2551, 2012.

[24] M. Benchohra and B. A. Slimani, "Existence and uniqueness of solutions to impulsive fractional differential equations," Electronic Journal of Differential Equations, vol. 2009, no. 10, pp. 1-11, 2009.
[25] D. J. Guo, "Existence and uniqueness of positive fixed points for mixed monotone operators and applications," Applicable Analysis, vol. 46, no. 1-2, pp. 91-100, 1992.

[26] D. J. Guo and V. Lakshmikantham, Nonlinear Problems in Abstract Cones, vol. 5 of Notes and Reports in Mathematics in Science and Engineering, Academic Press, Boston, Mass, USA, 1988. 


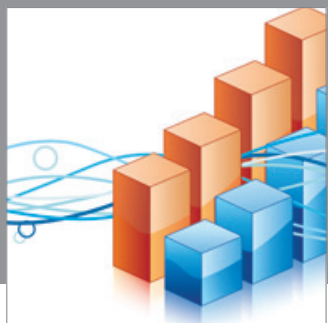

Advances in

Operations Research

mansans

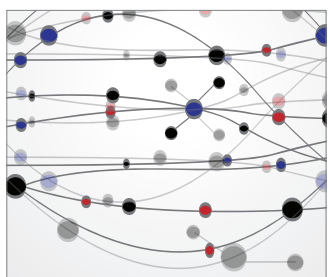

The Scientific World Journal
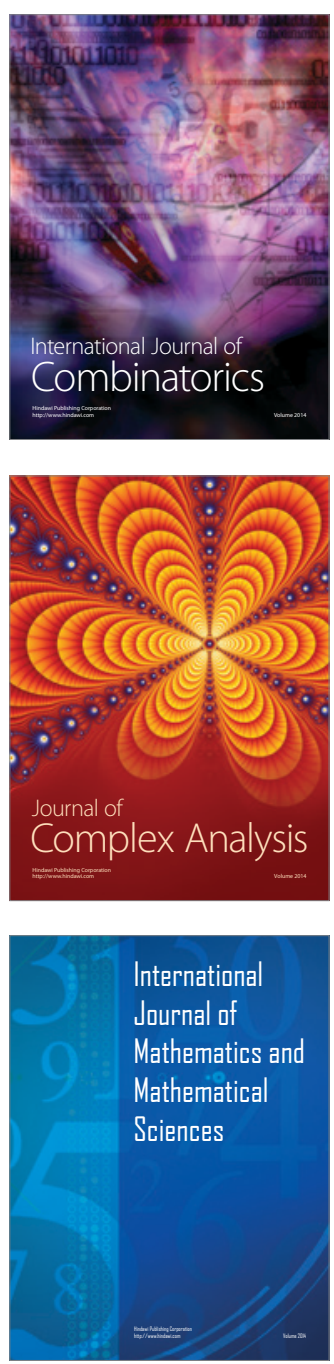
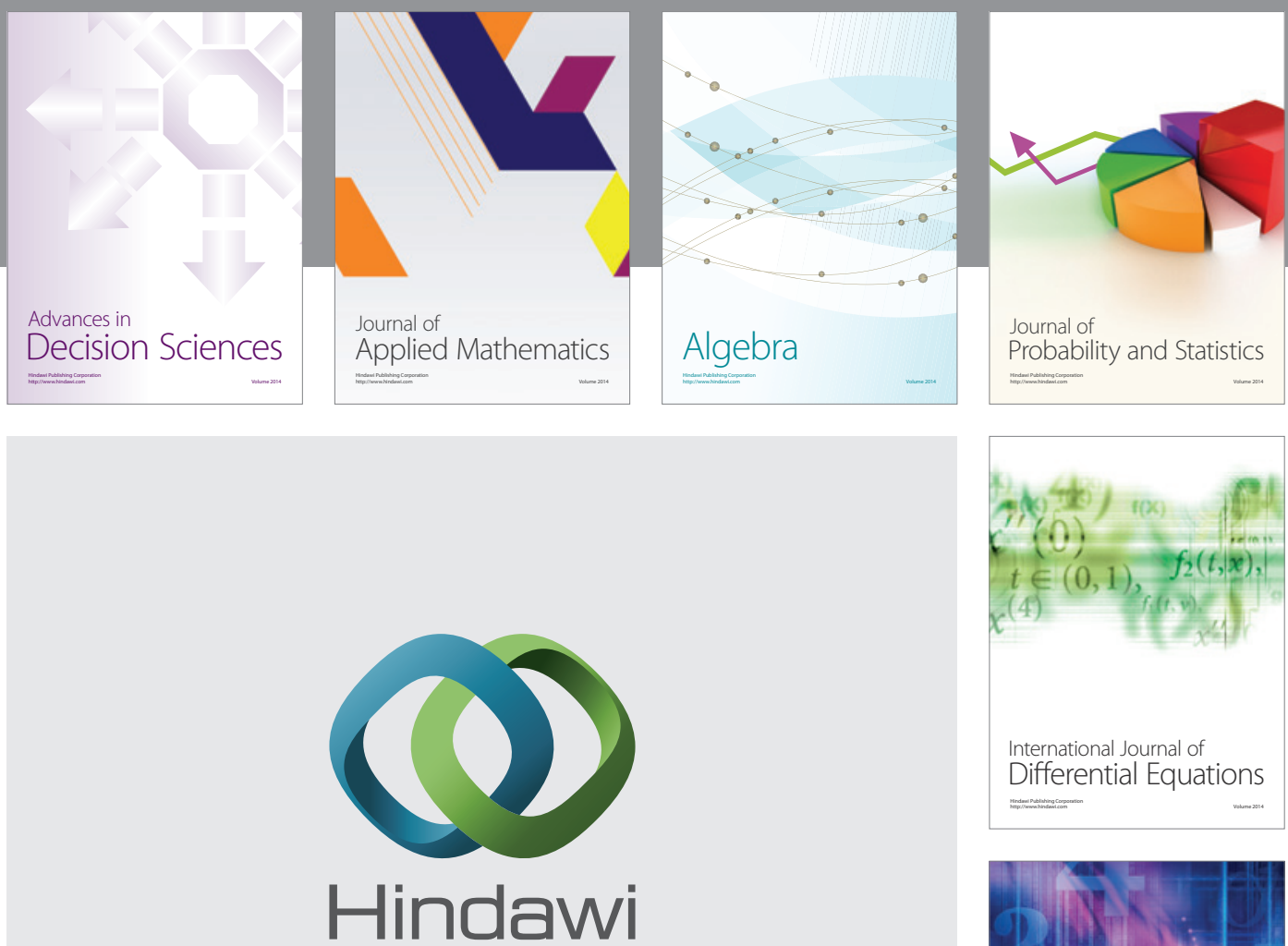

Submit your manuscripts at http://www.hindawi.com
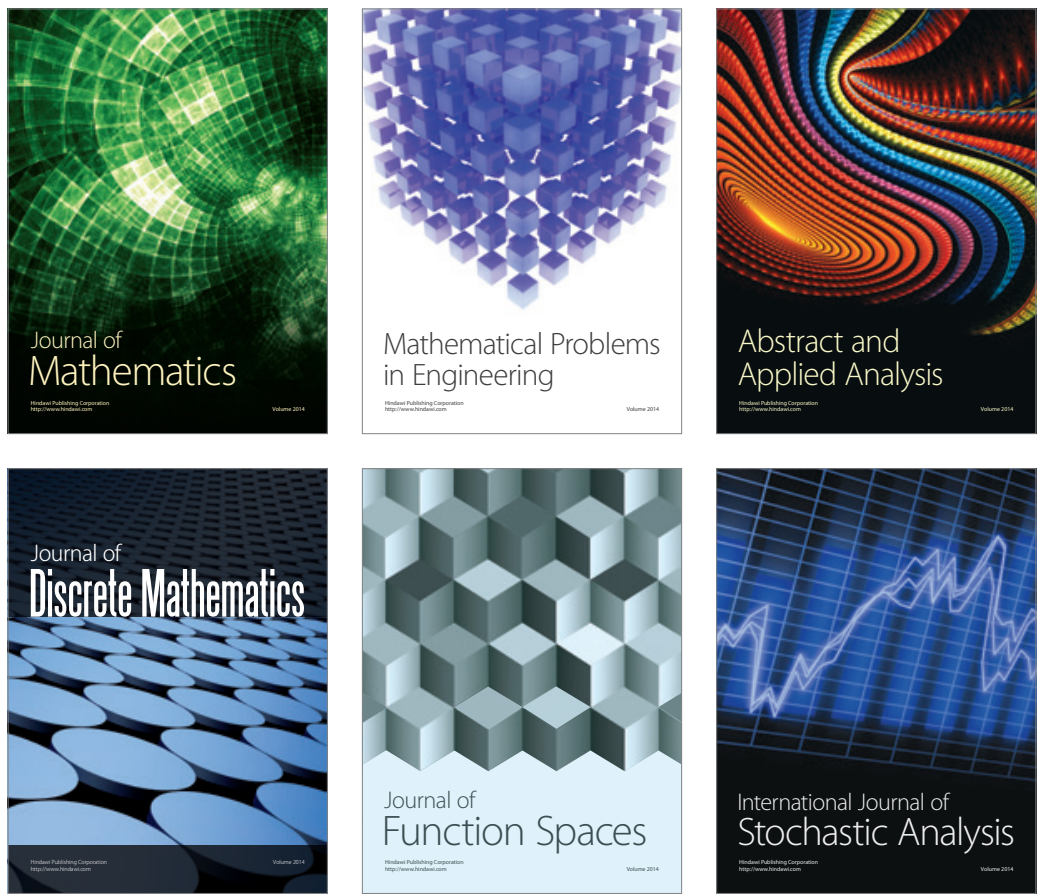

Journal of

Function Spaces

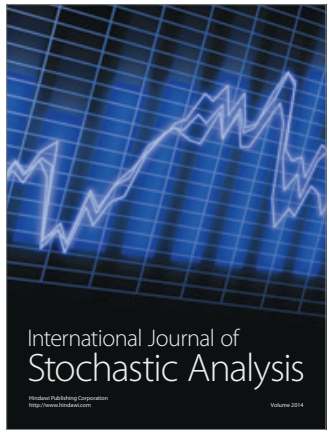

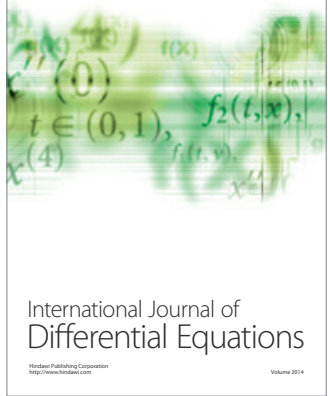
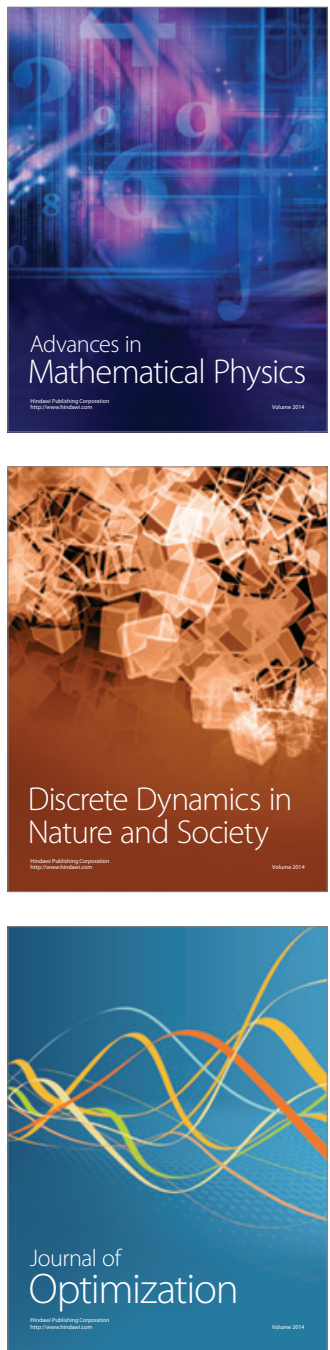